near the seturation limit.

(3) For stimuli of medium intensity, i. e. those producing responses corresponding to the linear portion of the curve representing intensity of responses against that of stimuli, (a) characteristics depend as much on the frequency as the audibility threshold of the human ear, (b) contours representing the equal response voltage levels in the response voltage-stimulus frequency. chart run along the course reminding of the contours of the equal loudness levels in the human audiogram.

(4) For medium stimulating pressure the wave form of the stimulating sound is reproduced in the electric cochlear response. But for low tones, especially of high intensities, the wave form of the response is markedly distorted.

(5) The acoustic nerve response was also observed in guinea-pigs stimulated at $100 \mathrm{cps}$. It showed a higher noise level and a shorter linear functional range in the stimulus-response relation than the corresponding cochlear response.

(6) When observation of the electric cocblear response or its change in the characteristics is intended for the purpose of examining the function of transmission system of the ear, the observation ought to be made over a sufficiently wide range so as to cover the whole stretch of the characteristic curve repre. senting intensity relation of response against the stimulus. Or if the restriction of the test range is desired, observation ought to be directed to the linear functional portion especially its median value of the characteristic curve.

\title{
EXPERIMENTAL STUDY ON THE TRANSMISSION OF MECHANICAL VIBRATION BY THE CONDUCTION SYSTEM OF THE MIDDLE EAR BY MEANS OF ELECTRIC COCHLEAR RESPONSE METHOD. APPARATUS AND METHOD OF OBSERVATION.
}

By

\section{J. UKIDA}

From the Department of Otorhinolaryngology, Okayama Medical College (Director: Professor D. Oda, and later Professor S. Takahara).

Main feature of the construction of the experimental apparatus:

(a) Sound source-Transitron circuit beat frequncy oscillator showing the excellent wave forms in the frequency range from 50 c. p. s. to $20 \mathrm{Kc}$. p. s., served as the source. As sound generator loud speaker or dynamic receiver, both calibrated, was used. 
(b) Amplifier was of R-C coupled type with high gain and excellent frequency characteristics.

(c) Response measurement was made with a valve voltmeter and a cathode ray oscillograph.

All parts of the system were prevented from the influence of useless mechanical vibration and shielded from interfering electric and acoustic distur. bances. Errors due to artefact were carefully avoided. The lowest measureable response voltage was $2-5$ microvolts and the distinctness of syllable articulation was tested after Logadorm. As the sound stimuli, seven serial octave tones (128-8192 c.p.s.), were generally used combined with an acustic low pass filter to obtain pure tones.

\section{ON THE ALBUMIN/GLOBULIN RATIO OF BLOOD SERUM IN RABBITS SHOWING DOWNWARD POSTURAL NYSTAGMUS. FIRST NOTE By K. IZUMI}

\section{From the Oto-Rhino-Laryngological Department, Osaka Municipal College of Medicine.}

A series of experiments were carried out to elucidate physiological mechanism involved in the downward positional nystagmus (Hasegawa) observed in ganglionectomized rabbits. Preliminary observation revealed the following:

1. Determination of $A / G$ ratio by salting out with $28 \mathrm{~g} / \mathrm{dl}$ solution of $\mathrm{Na}_{\mathrm{SO}} \mathrm{SO}_{3}$ is markedly affected by the temperature. The author controlled the temperature at $30.0^{\circ} \mathrm{C}$.

2. The positional nystagmus elicited in ganglionectomized rabbits is directed obliquely downwad as referred to the body axis of the rabbit.

3. Occurrence of this type of nystagmus after the ganglionectomy is the same between the unilateral and the bilateral section.

4. Appearance of this nystagmus persists 1 to 3 weeks.

5. A/G ratio of the serm, of rabbits or human, statistically showed a normal distribution of frequencies. 